doi:10.13108/2020-12-3-69

\title{
ON ANTIPERIODIC BOUNDARY VALUE PROBLEM FOR SEMILINEAR FRACTIONAL DIFFERENTIAL INCLUSION WITH DEVIATING ARGUMENT IN BANACH SPACE
}

\author{
G.G. PETROSYAN
}

\begin{abstract}
We consider a boundary value problem for a semi-linear differential inclusion of Caputo fractional derivative and a deviating coefficient in a Banach space. We assume that the linear part of the inclusion generates a bounded $C_{0}$-semigroup. A nonlinear part of the inclusion is a multi-valued mapping depending on the time and the prehistory of the function before a current time. The boundary condition is functional and anti-periodic in the sense that one function is equals to another with an opposite sign. To solve the considered problem, we employ the theory of fractional mathematical analysis, the properties of Mittag-Leffler as well as the theory of topological power for multi-valued condensing maps. The idea is as follws: the original problem is reduced to the existence of fixed points of a corresponding resolving multi-valued integral operator in the space of continuous functions. To prove the existence of the fixed points of the resolving multi-operator we employ a generalized theorem of Sadovskii type on a fixed point. This is why we show that the resolving integral multi-operator is condensing with respect to a vector measure of non-compactness in the space of continuous functions and maps a closed ball in this space into itself.
\end{abstract}

Keywords: Caputo fractional derivative, semi-linear differential inclusion, boundary value problem, fixed point, condensing multi-mapping, measure of non-compactness.

Mathematics Subject Classification: 34G25, 34K09, 34K37, 47H04, 47H08, 47H10

\section{INTRODUCTION}

The study of controlled systems with nonlinear components is a complicated and extremely important part of the modern mathematical control theory and harmonic analysis, with many applications, attracting a lot of attention both in Russia and entire world. The developing of the theory of differential inclusions is due to the fact that they are a natural and convenient approach for describing controlled systems from various classes, systems with discontinuous characteristics studied in various parts of the optimal control theory, mathematical physics, radiophysics, acoustics, etc. However, solving of problems in the framework of the present theories is often a complicated problem since many of them have an adequate description in terms of differential equations and inclusions with fractional derivatives. Many physical, economical, biological and engineering problems, first of all related with processes in dynamical systems, lead one to boundary value problems for differential equations and inclusions of fractional order, see monographs [8], [16], [19], 20], 23] and paper [17]. During recent years, a large set of problems related with equations and inclusions of fractional order is very intensively studied in Russia and abroad, see paper [1]-[5], [10]-[15], [21], [22].

G.G. Petrosyan, On antiperiodic Boundary VAlue Problem For SEMilinear Fractional DifFERENTIAL INCLUSION With DEVIATING ARGUMENT IN BANACH SPACE.

(c) G.G. Petrosyan G.G. 2020.

The reported study was funded by RFBR according to the research project no. 19-31-60011.

Submitted January 16, 2020. 
In the present work we study the solvability of a boundary value problem for a semi-linear differential inclusion of a fractional order in a separable Banach space $E$ :

$$
{ }^{C} D^{q} x(t) \in A x(t)+F\left(t, x_{t}\right), \quad t \in[0, T],
$$

with an antiperiodic boundary condition

$$
x_{0} \equiv-x_{T} .
$$

Here the symbol ${ }^{C} D^{q}$ stands for the Caputo fractional derivative of order $q \in(0,1), A: D(A) \subset$ $E \rightarrow E$ is a linear operator generating a bounded $C_{0}$-semigroup; the details on the theory of operator semigroups can be found in monograph [6], $F:[0, T] \times C([-h, 0] ; E) \multimap E$ is a nonlinear multivalued mapping and the function $x_{t}$ describes the prehistory of the solution up to the moment $t \in[0, T]$, that is, $x_{t}(s)=x(t+s), s \in[-h, 0], 0<h<T$, and we suppose that $x_{0}, x_{T} \in C([-h, 0] ; E)$. To solve this problem, we shall make use of the theory of topological degree for multi-valued condensing mappings. The idea of solving is as follows: the original problem is reduced to the problem on existence of fixed points for a corresponding resolving multi-valued integral operator. To prove the existence of fixed points of the resolving multioperator, we shall employ a generalized fixed point theorem of Sadovskii type.

\section{PRELIMINARIES}

2.1. Fractional integral and fractional derivative. We first introduce needed notions and notation from fractional mathematical analysis, more details can be found in monographs [16], [19], [23].

Let $E$ be a real Banach space.

Definition 2.1. A fractional integral of order $q \in(0,1)$ of a function $g:[0, T] \rightarrow E$ is a function $I^{q} g$ of the following form:

$$
I^{q} g(t)=\frac{1}{\Gamma(q)} \int_{0}^{t}(t-s)^{q-1} g(s) d s,
$$

where $\Gamma$ is the Euler Gamma function:

$$
\Gamma(q)=\int_{0}^{\infty} x^{q-1} e^{-x} d x
$$

Definition 2.2. A Riemann-Liouville fractional derivative of order $q \in(0,1)$ of a continuous function $g:[0, T] \rightarrow E$ is a function $D^{q} g$ of the following form:

$$
D^{q} g(t)=\frac{1}{\Gamma(1-q)} \quad \frac{d}{d t} \int_{0}^{t}(t-s)^{-q} g(s) d s
$$

provided the right hand side is well-defined.

Definition 2.3. A Caputo fractional derivative of order $q \in(N-1, N]$ of a function $g \in C^{N}([0, T] ; E)$ is a function ${ }^{C} D_{0}^{q} g$ of the following form:

$$
{ }^{C} D_{0}^{q} g(t)=\frac{1}{\Gamma(N-\alpha)} \int_{0}^{t}(t-s)^{N-q-1} g^{(N)}(s) d s
$$

provided the right hand side is well-defined. 
A Caputo fractional derivative of order $q \in(0,1)$ of a continuous function $g:[0, T] \rightarrow E$ is related with a Riemann-Liouville fractional derivative of order $q \in(0,1)$ by the following formula:

$$
{ }^{C} D^{q} g(t)=\left(D^{q}(g(\cdot)-g(0))\right)(t) .
$$

A great advantage of the Caputo fractional derivative, in comparison with a RiemannLiouville fractional derivative, is that the former keeps all main properties of the derivatives of integer order, for instance, the vanishing of the derivative of a constant.

Definition 2.4. A function of form

$$
E_{q, \beta}(z)=\sum_{n=0}^{\infty} \frac{z^{n}}{\Gamma(q n+\beta)}, \quad q, \beta>0, \quad z \in \mathbb{C},
$$

is called a Mittag-Leffler function.

As a rule, the function $E_{q, 1}$ is denoted simply by $E_{q}$. The Mittag-Leffler function plays an important role in the fractional calculus. For instance, consider the Cauchy problem for a scalar fractional differential equation:

$$
\begin{aligned}
& { }^{C} D^{q} x(t)=\lambda x(t)+f(t), \quad t \in[0, T], \\
& x(0)=x_{0},
\end{aligned}
$$

where $\lambda \in \mathbb{R}$ and $f:[0, T] \rightarrow \mathbb{R}$ is a continuous function. A solution to this problem is a continuous function $x:[0, T] \rightarrow \mathbb{R}$ satisfying condition $(2.2)$, for which the Caputo fractional derivative ${ }^{C} D^{q} x$ is also continuous and satisfies equation 2.1]. It is known, see [16, Exam. 4.9] that the only solution to this problem is the function

$$
x(t)=E_{q}\left(\lambda t^{q}\right) x_{0}+\int_{0}^{t}(t-s)^{q-1} E_{q, q}\left(\lambda(t-s)^{q}\right) f(s) d s .
$$

Below we shall make use of the following relations [7]:

$$
\begin{aligned}
& E_{q, \beta}(z)=\frac{1}{\Gamma(\beta)}+z E_{q, \beta+q}(z), \\
& \int_{0}^{z} t^{\beta-1} E_{q, \beta}\left(\lambda t^{q}\right) d t=z^{\beta} E_{q, \beta+1}\left(\lambda z^{q}\right) .
\end{aligned}
$$

2.2. Measures of non-compactness and condensing mappings. Let $\mathcal{E}$ be a Banach space. We introduce the following notations:

- $P(\mathcal{E})=\{A \subseteq \mathcal{E}: A \neq \varnothing\}$;

- $P b(\mathcal{E})=\{A \in P(\mathcal{E}): A$ is bounded $\}$;

- $P v(\mathcal{E})=\{A \in P(\mathcal{E}): A$ is convex $\}$;

- $K(\mathcal{E})=\{A \in P b(\mathcal{E}): A$ is compact $\}$;

- $K v(\mathcal{E})=P v(\mathcal{E}) \cap K(\mathcal{E})$.

Definition 2.5 (see, for instance, [9]). Let $(\mathcal{A}, \geqslant)$ be a partially ordered set. The function $\beta: P b(\mathcal{E}) \rightarrow \mathcal{A}$ is a called a measure of non-compactness $(M N C)$ in $\mathcal{E}$ if for each $\Omega \in P b(\mathcal{E})$ the identity

$$
\beta(\overline{\mathrm{co}} \Omega)=\beta(\Omega)
$$

holds true, where $\overline{\mathrm{co}} \Omega$ denotes the closure of the convex hull of $\Omega$.

A measure of non-compactness $\beta$ is called 
1) monotonous if for each $\Omega_{0}, \Omega_{1} \in P b(\mathcal{E})$, the embedding $\Omega_{0} \subseteq \Omega_{1}$ implies the inequality $\beta\left(\Omega_{0}\right) \leqslant \beta\left(\Omega_{1}\right)$

2) non-singular if for each $a \in \mathcal{E}$ and each $\Omega \in P b(\mathcal{E})$ the identity $\beta(\{a\} \cup \Omega)=\beta(\Omega)$ holds.

If $\mathcal{A}$ is a cone in a Banach space, then a measure of non-compactness $\beta$ is called

3) regular if the identity $\beta(\Omega)=0$ is equivalent to a relative compactness of the set $\Omega \in$ $\operatorname{Pb}(\mathcal{E})$

4) real if $\mathcal{A}$ is a subset of real numbers $\mathbb{R}$ with the natural order;

5) algebraically semi-additive if $\beta\left(\Omega_{0}+\Omega_{1}\right) \leqslant \beta\left(\Omega_{0}\right)+\beta\left(\Omega_{1}\right)$ for all $\Omega_{0}, \Omega_{1} \in P b(\mathcal{E})$.

An example of a real MNC obeying all aforementioned properties is Hausdorff MNC $\chi(\Omega)$ :

$$
\chi(\Omega)=\inf \{\varepsilon>0 \text { for which } \Omega \text { possesses a finite } \varepsilon \text {-net in } \mathcal{E}\} .
$$

We observe that the Hausdorff MNC satisfies also semi-homogeneity property:

$$
\chi(\lambda \Omega)=|\lambda| \chi(\Omega),
$$

for all $\lambda \in \mathbb{R}$ and $\Omega \in P(\mathcal{E})$. Moreover, if $\mathcal{L}: \mathcal{E} \rightarrow \mathcal{E}$ is a linear bounded operator, then

$$
\chi(\mathcal{L}(\Omega))=\|\mathcal{L}\| \chi(\Omega)
$$

for each $\Omega \in P(\mathcal{E})$.

A norm of a set $M \in P b(\mathcal{E})$ is defined by the formula:

$$
\|M\|=\sup _{x \in M}\|x\|_{\mathcal{E}} .
$$

2.3. Multi-valued mappings. The following notions and statements can be found in monographs [9], [18].

Definition 2.6. Let $X$ be a closed subset in $\mathcal{E}$ and $\beta$ be a $M N C$ in $\mathcal{E}$. A multi-valued mapping (multi-mapping) $\mathcal{F}: X \rightarrow K(\mathcal{E})$ is called condensing with respect to the $M N C \beta$ (or $\beta$-condensing) if for each $\Omega \in P b(X)$ not being relatively compact a relation holds:

$$
\beta(F(\Omega)) \ngtr \beta(\Omega) .
$$

Definition 2.7. Let $X$ be a metric space. A multi-mapping $\mathcal{F}: X \rightarrow P(\mathcal{E})$ is called lower semi-continuous if

$$
\mathcal{F}^{-1}(V)=\{x \in X: \mathcal{F}(x) \subset V\}
$$

is an open subset in $X$ for each open set $V \subset \mathcal{E}$.

Theorem 2.1 ([9, Cor. 3.3.1]). Let $\mathcal{M}$ be a bounded convex closed subset $\mathcal{E}$ and $\mathcal{F}: \mathcal{M} \rightarrow$ $K v(\mathcal{M})$ be an upper semi-continuous $\beta$-condensing multi-mapping, where $\beta$ is a non-singular $M N C$ in $\mathcal{E}$. Then the set of fixed points Fix $\mathcal{F}=\{x: x \in \mathcal{F}(x)\}$ is a non-empty set.

Definition 2.8. For $1 \leqslant p \leqslant \infty$, a multi-function $G:[0, \tau] \rightarrow K(\mathcal{E})$ is called

- $L^{p}$-integrable if it admits a Bochner $L^{p}$-integrable selection, that is, there exists a function $g \in L^{p}((0, \tau) ; \mathcal{E})$ such that $g(t) \in G(t)$ for almost each $t \in[0, \tau]$;

- $L^{p}$-integrally bounded if there exists a function $\xi \in L^{p}((0, \tau))$ such that

$$
\|G(t)\| \leqslant \xi(t)
$$

for almost each $t \in[0, \tau]$.

The set of $L^{p}$-integrable selections of a multi-function $G:[0, \tau] \rightarrow K(\mathcal{E})$ is denoted by $\mathcal{S}_{G}^{p}[0, \tau]$.

Definition 2.9. An integral for an $L^{p}$-integrable multi-function $G:[0, \tau] \rightarrow K(\mathcal{E})$ is defined as follows:

$$
\int_{0}^{\tau} G(s) d s=\left\{\int_{0}^{\tau} f(s) d s: f \in \mathcal{S}_{G}^{p}[0, \tau]\right\} .
$$


Lemma 2.1 ([9, Thm. 4.2.3]). Let $\mathcal{E}$ be a separable Banach space and $G:[0, \tau] \rightarrow K(\mathcal{E})$ be an integrable, integrally bounded multi-function such that

$$
\chi(G(t)) \leqslant v(t) \quad \text { for a.e. } t \in[0, \tau],
$$

where $\chi$ is the Hausdorff $M N C$ in $\mathcal{E}$ and $v \in L_{+}^{1}(0, \tau)$. Then

$$
\chi\left(\int_{0}^{\tau} G(s) d s\right) \leqslant \int_{0}^{\tau} v(s) d s .
$$

\section{EXISTENCE OF SOLUTIONS}

In what follows, for the sake of brevity we denote $\mathcal{C}:=C([-h, 0] ; E)$.

We assume that the operator $A$ obeys a condition:

(A) $A: D(A) \subseteq E \rightarrow E$ is a linear closed (not necessarily bounded) operator generating a bounded $C_{0}$-semigroup $\{U(t)\}_{t \geqslant 0}$ of linear operators in $E$.

We impose the following conditions for the multi-valued operator $F:[0, T] \times \mathcal{C} \rightarrow K v(E)$ :

$(F 1)$ for each $\xi \in \mathcal{C}$, a multi-function $F(\cdot, \xi):[0, T] \rightarrow K v(E)$ admits a measurable selection;

$(F 2)$ for almost each $t \in[0, T]$, the multi-operator $F(t, \cdot): E \rightarrow K v(E)$ is upper semicontinuous;

(F3) there exists a function $\alpha \in L_{+}^{\infty}([0, T])$ such that for each $\xi \in \mathcal{C}$ the inequality

$$
\|F(t, \xi)\|_{E} \leqslant \alpha(t)\left(1+\|\xi\|_{\mathcal{C}}\right) \quad \text { for a.e. } t \in[0, T]
$$

holds;

(F4) there exists a function $\mu \in L_{+}^{\infty}([0, T])$ such that for each bounded set $\Delta \subset \mathcal{C}$, the inequality

$$
\chi(F(t, \Delta)) \leqslant \mu(t) \varphi(\Delta)
$$

holds for almost each $t \in[0, T]$, where $\varphi(\Delta)=\sup _{s \in[-h, 0]} \chi(\Delta(s)), \chi$ is the Hausdorff MNC in $E$ and $\Delta(s)=\{y(s): y \in \Delta\}$.

For a function $x \in C([-h, T] ; E)$ we consider a multi-function

$$
\Phi:[0, T] \rightarrow K v(E), \quad \Phi(t)=F\left(t, x_{t}\right) .
$$

It follows from conditions (F1)-(F3) [9, Thm. 1.3.5] that a multi-function $\Phi$ is $L^{\infty}$-integrable and this is why a superposition multi-operator $\mathcal{P}_{F}^{\infty}: C([-h, T] ; E) \rightarrow P\left(L^{\infty}([0, T] ; E)\right)$ can be defined as follows:

$$
\mathcal{P}_{F}^{\infty}(x)=\mathcal{S}_{\Phi}^{\infty}[0, T] .
$$

Definition 3.1. A mild solution to inclusion (1.1) is a function $x \in C([-h, T] ; E)$ satisfying the identity

$$
x(t)=\mathcal{G}(t) x(0)+\int_{0}^{t}(t-s)^{q-1} \mathcal{T}(t-s) \phi(s) d s, \quad t \in[0, T],
$$

where $\phi \in \mathcal{P}_{F}^{\infty}(x)$,

$$
\begin{aligned}
& \mathcal{G}(t)=\int_{0}^{\infty} \xi_{q}(\theta) U\left(t^{q} \theta\right) d \theta, \mathcal{T}(t)=q \int_{0}^{\infty} \theta \xi_{q}(\theta) U\left(t^{q} \theta\right) d \theta \\
& \xi_{q}(\theta)=\frac{1}{q} \theta^{-1-\frac{1}{q}} \Psi_{q}\left(\theta^{-1 / q}\right), \\
& \Psi_{q}(\theta)=\frac{1}{\pi} \sum_{n=1}^{\infty}(-1)^{n-1} \theta^{-q n-1} \frac{\Gamma(n q+1)}{n !} \sin (n \pi q), \theta \in \mathbb{R}_{+} .
\end{aligned}
$$


Remark 3.1 (see, for instance, [21], [23]). The following relations hold:

$$
\xi_{q}(\theta) \geqslant 0, \quad \int_{0}^{\infty} \xi_{q}(\theta) d \theta=1, \quad \int_{0}^{\infty} \theta \xi_{q}(\theta) d \theta=\frac{1}{\Gamma(q+1)} .
$$

Remark 3.2 (see, for instance, [17]). In the scalar case when $E=\mathbb{R}$ and $U(t)=e^{-\eta t}$ with $\eta>0$ we have:

$$
\mathcal{G}(t)=E_{q}\left(-\eta t^{q}\right), \quad \mathcal{T}(t)=E_{q, q}\left(-\eta t^{q}\right), \quad t \in[0, T] .
$$

This is why the following identities hold:

$$
E_{q}(-z)=\int_{0}^{\infty} \xi_{q}(\theta) e^{-z \theta} d \theta, \quad E_{q, q}(-z)=\int_{0}^{\infty} q \theta \xi_{q}(\theta) e^{-z \theta} d \theta,
$$

which imply that

$$
E_{q}(\tau)>0, \quad E_{q, q}(\tau)>0 \text { for } \quad \tau<0 .
$$

Lemma 3.1 (see [23], 21]). The operator functions $\mathcal{G}$ and $\mathcal{T}$ satisfy the following conditions:

1) for each $t \in[0, T], \mathcal{G}(t)$ and $\mathcal{T}(t)$ are linear bounded operators and moreover, if the semigroup $U(t)$ satisfies the estimate

$$
\|U(t)\| \leqslant e^{-\eta t}, \quad t \geqslant 0
$$

with $\eta>0$, then

$$
\begin{aligned}
& \|\mathcal{G}(t)\| \leqslant E_{q}\left(-\eta t^{q}\right) \leqslant 1, \quad t \in[0, T] \\
& \|\mathcal{T}(t)\| \leqslant E_{q, q}\left(-\eta t^{q}\right) \leqslant \frac{q}{\Gamma(1+q)}, \quad t \in[0, T]
\end{aligned}
$$

2) the operator functions $\mathcal{G}(\cdot)$ and $\mathcal{T}(\cdot)$ are strongly continuous, that is, the functions $t \in$ $[0, T] \rightarrow \mathcal{G}(t) x$ and $t \in[0, T] \rightarrow \mathcal{T}(t) x$ are continuous for all $x \in E$.

To solve the formulated problem, we assume the following condition:

$$
1 \notin s p[-\mathcal{G}(T)] \text {. }
$$

We consider a multi-operator $G: C([-h, T] ; E) \rightarrow P(C([-h, T] ; E))$ defined as

$$
G(x)=\{y\}
$$

for all functions $y$ of form

$$
\left\{\begin{aligned}
y(t)= & -\mathcal{G}(t)(I+\mathcal{G}(T))^{-1} \int_{0}^{T}(T-s)^{q-1} \mathcal{T}(T-s) \phi(s) d s \\
& +\int_{0}^{t}(t-s)^{q-1} \mathcal{T}(t-s) \phi(s) d s, \quad t \in[0, T], \\
y(s)= & -y(T-s), \quad s \in[-h, 0],
\end{aligned}\right.
$$

where $\phi \in \mathcal{P}_{F}^{\infty}(x)$.

The multi-operator $G$ is well-defined thanks to the following lemma.

Lemma 3.2. If $y \in G(x)$ for $x \in C([-h, T] ; E)$, then $y(0)=-y(T)$ and this is why $y_{0}=$ $-y_{T}$. 
Proof. Indeed,

$$
\begin{aligned}
y(T) & =-\mathcal{G}(T)(I+\mathcal{G}(T))^{-1} \int_{0}^{T}(T-s)^{q-1} \mathcal{T}(T-s) \phi(s) d s+\int_{0}^{T}(T-s)^{q-1} \mathcal{T}(T-s) \phi(s) d s \\
& =\left(-\mathcal{G}(T)(I+\mathcal{G}(T))^{-1}+I\right) \int_{0}^{T}(T-s)^{q-1} \mathcal{T}(T-s) \phi(s) d s \\
& =(I+\mathcal{G}(T))^{-1} \int_{0}^{T}(T-s)^{q-1} \mathcal{T}(T-s) \phi(s) d s=-y(0) .
\end{aligned}
$$

The identity $y_{0}=-y_{T}$ is implied by the definition of $G$. The proof is complete.

Theorem 3.1. Fixed points of the multi-operator $G$ are integral solutions of problem (1.1) (1.2) and vice versa, mild solutions of problem (1.1)-(1.2) are fixed points of the multi-operator G.

Proof. Let $x$ be a mild solution of problem (1.1)-(1.2), then for $t \in[0, T]$ the identity

$$
x(t)=\mathcal{G}(t) x(0)+\int_{0}^{t}(t-s)^{q-1} \mathcal{T}(t-s) \phi(s) d s
$$

holds true, where $\phi \in \mathcal{P}_{F}^{\infty}(x)$. Then it follows from Condition 1.2 that

$$
x(0)=-x(T)=-\mathcal{G}(T) x(0)-\int_{0}^{T}(T-s)^{q-1} \mathcal{T}(T-s) \phi(s) d s,
$$

which implies the identity

$$
x(0)=-(I+\mathcal{G}(T))^{-1} \int_{0}^{T}(T-s)^{q-1} \mathcal{T}(T-s) \phi(s) d s .
$$

Thanks to this identity, for $t \in[0, T]$ we obtain:

$$
x(t)=-\mathcal{G}(t)(I+\mathcal{G}(T))^{-1} \int_{0}^{T}(T-s)^{q-1} \mathcal{T}(T-s) \phi(s) d s+\int_{0}^{t}(t-s)^{q-1} \mathcal{T}(t-s) \phi(s) d s
$$

and therefore, $x \in \operatorname{Fix} G$.

Vice versa, let $x \in \operatorname{Fix} G$, then for $t \in[0, T]$ this function solves equation (3.8) with $\phi \in$ $\mathcal{P}_{F}^{\infty}(x)$. This implies that this function is a mild solution of inclusion (1.1). In its turn, the validity of condition $(1.2$ follows from Lemma 3.2 . The proof is complete.

We proceed to studying topological properties of the multi-operator $G$.

Lemma 3.3. The multi-operator $G$ is upper semi-continuous and has compact values.

Proof. It is obvious that it is sufficient to prove the statement for the restriction of the multioperator $G$ on the space $C([0, T] ; E)$. We denote this restriction by $\widetilde{G}$.

The multi-operator $\widetilde{G}: C([-h, T] ; E) \rightarrow P(C([0, T] ; E))$ can be represented via superpositions:

$$
\widetilde{G}(x)=\sigma \circ \bar{e} \circ \bar{g} \circ S \circ \mathcal{P}_{F}^{\infty}(x)
$$


where

$$
\begin{aligned}
& S: L^{\infty}([0, T] ; E) \rightarrow C([0, T] ; E), \\
& S(\phi)(t)=\int_{0}^{t}(t-s)^{q-1} \mathcal{T}(t-s) \phi(s) d s, \\
& \bar{g}: C([0, T] ; E) \rightarrow C([0, T] ; E) \times C([0, T] ; E), \\
& \bar{g}(u)=(u, u), \\
& \bar{e}: C([0, T] ; E) \times C([0, T] ; E) \rightarrow C([0, T] ; E) \times C([0, T] ; E), \\
& \bar{e}(u, v)=(w, v), \\
& w(t)=-\mathcal{G}(t)(I+\mathcal{G}(T))^{-1} u(T), \\
& \sigma: C([0, T] ; E) \times C([0, T] ; E) \rightarrow C([0, T] ; E), \\
& \sigma(u, v)=u+v .
\end{aligned}
$$

It was proved in work [10] that the multi-operator $S \circ \mathcal{P}_{F}^{\infty}$ is upper semi-continuous and has compact values. Taking into consideration that $\bar{g}, \bar{e}$ and $\sigma$ are linear bounded operators, we obtain the desired result. The proof is complete.

We are going to show that the multi-operator $G$ is condensing; in order to do this, we consider the cone

$$
\mathbb{R}_{+}^{2}=\left\{\zeta=\left(\zeta_{1}, \zeta_{2}\right): \zeta_{1} \geqslant 0, \zeta_{2} \geqslant 0\right\}
$$

treating $\mathbb{R}_{+}$as a linear ordered set with the natural order. In the space $C([-h, T] ; E)$ we introduce an MNC

defined as

$$
\nu: P(C([-h, T] ; E)) \rightarrow \mathbb{R}_{+}^{2}
$$

$$
\nu(\Omega)=\left(\varphi(\Omega), \bmod _{C}(\Omega)\right),
$$

where $\varphi(\Omega)$ is the modulus of the fiber non-compactness:

$$
\varphi(\Omega)=\sup _{t \in[-h, T]} \chi(\{y(t): y \in \Omega\}),
$$

while the second component of the modulus of the equicontinuity:

$$
\bmod _{C}(\Omega)=\lim _{\delta \rightarrow 0} \sup _{u \in \Omega} \max _{\left|t_{1}-t_{2}\right| \leqslant \delta}\left\|u\left(t_{1}\right)-u\left(t_{2}\right)\right\| .
$$

Theorem 3.2. Under conditions $(A),(F 1)-(F 4)$ and the condition (A1) the semi-group $U$ obeys estimate (3.3) for some $\eta>0$. If

$$
\frac{\|\mu\|_{\infty}}{\eta}<1
$$

where $\mu(\cdot)$ is the function from condition (F4), then the multi-operator $G$ is $\nu$-condensing.

Proof. Let $\Omega \subset C([-h, T] ; E)$ be a non-empty bounded set, for which

$$
\nu(G(\Omega)) \geqslant \nu(\Omega) .
$$

We are going to show that $\Omega$ is a relatively compact set.

It follows from $(3.12)$ that

$$
\varphi(G(\Omega)) \geqslant \varphi(\Omega) .
$$

Let $0 \leqslant t \leqslant T$. Employing estimates $(3.4)-(3.5)$, property (F4) and denoting

$$
\Omega_{s}=\left\{x_{s}: x \in \Omega\right\},
$$


for $0 \leqslant s \leqslant T, \Omega_{s} \subset \mathcal{C}$, we obtain:

$$
\begin{aligned}
\chi(G(\Omega)(t)) \leqslant & \chi\left(-\mathcal{G}(t)(I+\mathcal{G}(T))^{-1} \int_{0}^{T}(T-s)^{q-1} \mathcal{T}(T-s) F\left(s, \Omega_{s}\right) d s\right. \\
& \left.+\int_{0}^{t}(t-s)^{q-1} \mathcal{T}(t-s) F\left(s, \Omega_{s}\right) d s\right) \\
\leqslant & \|-\mathcal{G}(t)\|\left\|(I-(-\mathcal{G}(T)))^{-1}\right\| \chi\left(\int_{0}^{T}(T-s)^{q-1} \mathcal{T}(T-s) F\left(s, \Omega_{s}\right) d s\right) \\
& +\chi\left(\int_{0}^{t}(t-s)^{q-1} \mathcal{T}(t-s) F\left(s, \Omega_{s}\right) d s\right) \\
\leqslant & \frac{E_{q}\left(-\eta t^{q}\right)}{1-E_{q}\left(-\eta T^{q}\right)} \int_{0}^{T}(T-s)^{q-1} E_{q, q}\left(-\eta(T-s)^{q}\right) \mu(s) \varphi\left(\Omega_{s}\right) d s \\
& +\int_{0}^{t}(t-s)^{q-1} E_{q, q}\left(-\eta(t-s)^{q}\right) \mu(s) \varphi\left(\Omega_{s}\right) d s \\
\leqslant & \frac{E_{q}\left(-\eta t^{q}\right)}{1-E_{q}\left(-\eta T^{q}\right)}\|\mu\|_{\infty} \sup _{t \in[-h, T]} \chi(\Omega(t)) \int_{0}^{T}(T-s)^{q-1} E_{q, q}\left(-\eta(T-s)^{q}\right) d s \\
& +\|\mu\|_{\infty} \sup _{t \in[-h, T]} \chi(\Omega(t)) \int_{0}^{q}(t-s)^{q-1} E_{q, q}\left(-\eta(t-s)^{q}\right) d s .
\end{aligned}
$$

To proceed with estimating $\chi(G(\Omega)(t)), t \in[0, T]$, we calculate the integrals in the latter estimate by means of formula $(2.5)$ :

$$
\begin{aligned}
\int_{0}^{T}(T-s)^{q-1} E_{q, q}\left(-\eta(T-s)^{q}\right) d s & =-\int_{0}^{T}(T-s)^{q-1} E_{q, q}\left(-\eta(T-s)^{q}\right) d(T-s) \\
& =\int_{0}^{T} y^{q-1} E_{q, q}\left(-\eta y^{q}\right) d y=T^{q} E_{q, q+1}\left(-\eta T^{q}\right) .
\end{aligned}
$$

In the same way we obtain

$$
\int_{0}^{t}(t-s)^{q-1} E_{q, q}\left(-\eta(t-s)^{q}\right) d s=t^{q} E_{q, q+1}\left(-\eta t^{q}\right) .
$$

We note that choosing $\beta=1$ in formula 2.4 , we get:

$$
E_{q}\left(-\eta t^{q}\right)=\frac{1}{\Gamma(1)}-\eta t^{q} E_{q, q+1}\left(-\eta t^{q}\right)=1-\eta t^{q} E_{q, q+1}\left(-\eta t^{q}\right)
$$


Thus, we obtain the following identities:

$$
\begin{aligned}
& \int_{0}^{T}(T-s)^{q-1} E_{q, q}\left(-\eta(T-s)^{q}\right) d s=T^{q} \frac{1}{\eta T^{q}}\left(1-E_{q}\left(-\eta T^{q}\right)\right)=\frac{1}{\eta}\left(1-E_{q}\left(-\eta T^{q}\right)\right), \\
& \int_{0}^{t}(t-s)^{q-1} E_{q, q}\left(-\eta(t-s)^{q}\right) d s=\frac{1}{\eta}\left(1-E_{q}\left(-\eta t^{q}\right)\right) .
\end{aligned}
$$

This is why for $t \in[0, T]$ we have

$$
\begin{aligned}
\chi(G(\Omega)(t)) \leqslant & \frac{E_{q}\left(-\eta t^{q}\right)}{1-E_{q}\left(-\eta T^{q}\right)}\|\mu\|_{\infty} \sup _{t \in[-h, T]} \chi(\Omega(t)) \frac{1}{\eta}\left(1-E_{q}\left(-\eta T^{q}\right)\right) \\
& +\|\mu\|_{\infty} \sup _{t \in[-h, T]} \chi(\Omega(t)) \frac{1}{\eta}\left(1-E_{q}\left(-\eta t^{q}\right)\right) \\
= & \frac{\|\mu\|_{\infty}}{\eta} \sup _{t \in[-h, T]} \chi(\Omega(t)) .
\end{aligned}
$$

The latter estimate implies the inequality

$$
\sup _{t \in[0, T]} \chi(G(\Omega)(t)) \leqslant \frac{\|\mu\|_{\infty}}{\eta} \sup _{t \in[-h, T]} \chi(\Omega(t)) .
$$

At the same time, by the definition of the multi-operator $G$, the relation holds:

$$
\sup _{s \in[-h, 0]} \chi(G(\Omega)(s))=\sup _{t \in[T-h, T]} \chi(G(\Omega)(t)) \leqslant \sup _{t \in[0, T]} \chi(G(\Omega)(t)) .
$$

Taking into consideration estimates (3.14) and (3.15), we find that

$$
\sup _{t \in[-h, T]} \chi(G(\Omega)(t)) \leqslant \frac{\|\mu\|_{\infty}}{\eta} \sup _{t \in[-h, T]} \chi(\Omega(t)),
$$

or, equivalently,

$$
\varphi(G(\Omega)) \leqslant \frac{\|\mu\|_{\infty}}{\eta} \varphi(\Omega)
$$

It follows from the latter estimate and inequalities 3.11), 3.13) that

$$
\varphi(\Omega)=0 .
$$

It was shown in work [10] that on the segment $[0, T]$, the identity holds:

$$
\bmod _{C}\left(S \circ \mathcal{P}_{F}^{\infty}(\Omega)\right)=0 .
$$

For the multi-operator $\widetilde{G}$ in 3.9 we also obtain that

$$
\bmod _{C}(\widetilde{G}(\Omega))=0
$$

Employing the definition of the multi-operator $G$ once again, we get the identity:

$$
\bmod _{C}(G(\Omega))=0,
$$

which by 3.12 finally yields that

$$
\bmod _{C}(\Omega)=0 \text {. }
$$

By Arzelà-Ascoli theorem we obtain that $\Omega$ is a relatively compact set. The proof is complete.

Now we are in position to prove the main result of the work. 
Theorem 3.3. Under conditions (A), (A1), (F1)-(F4), if

$$
\frac{k}{\eta}<1
$$

where $k=\max \left\{\|\alpha\|_{\infty},\|\mu\|_{\infty}\right\}$, the functions $\alpha$ and $\mu$ are from conditions (F3) and (F4), respectively, $\eta$ is a constant from condition (A1), then problem (1.1)-(1.2) is solvable.

Proof. We choose arbitrary $x \in C([-h, T] ; E)$ and $y \in G(x)$, then for $\phi \in \mathcal{P}_{F}^{\infty}(x)$ and $t \in[0, T]$ we have the following estimate:

$$
\begin{aligned}
& \|y(t)\|_{E} \leqslant\left\|-\mathcal{G}(t)(I+\mathcal{G}(T))^{-1} \int_{0}^{T}(T-s)^{q-1} \mathcal{T}(T-s) \phi(s) d s+\int_{0}^{t}(t-s)^{q-1} \mathcal{T}(t-s) \phi(s) d s\right\|_{E} \\
& \leqslant\|-\mathcal{G}(t)\|\left\|(I-(-\mathcal{G}(T)))^{-1}\right\| \int_{0}^{T}(T-s)^{q-1} \mathcal{T}(T-s) \alpha(s)\left(1+\left\|x_{s}\right\|_{\mathcal{C}}\right) d s \\
& +\int_{0}^{t}(t-s)^{q-1}\|\mathcal{T}(t-s)\| \alpha(s)\left(1+\left\|x_{s}\right\|_{\mathcal{C}}\right) d s \\
& \leqslant \frac{E_{q}\left(-\eta t^{q}\right)}{1-E_{q}\left(-\eta T^{q}\right)} \int_{0}^{T}(T-s)^{q-1} E_{q, q}\left(-\eta(T-s)^{q}\right) \alpha(s)\left(1+\|x\|_{C([-h, T] ; E)}\right) d s \\
& +\int_{0}^{t}(t-s)^{q-1} E_{q, q}\left(-\eta(t-s)^{q}\right) \alpha(s)\left(1+\|x\|_{C([-h, T] ; E)}\right) d s \\
& \leqslant \frac{E_{q}\left(-\eta t^{q}\right)}{1-E_{q}\left(-\eta T^{q}\right)}\|\alpha\|_{\infty}\left(1+\|x\|_{C([-h, T] ; E)}\right) \frac{1}{\eta}\left(1-E_{q}\left(-\eta T^{q}\right)\right) \\
& +\|\alpha\|_{\infty}\left(1+\|x\|_{C([-h, T] ; E)}\right) \frac{1}{\eta}\left(1-E_{q}\left(-\eta t^{q}\right)\right) \\
& =\frac{\|\alpha\|_{\infty}}{\eta}\left(1+\|x\|_{C([-h, T] ; E)}\right) \leqslant \frac{k}{\eta}\left(1+\|x\|_{C([-h, T] ; E)}\right) .
\end{aligned}
$$

We note that by the definition of the multi-operator $G$, the latter estimate holds also for $t \in[-h, 0]$.

Now we take

$$
R \geqslant \frac{k \eta^{-1}}{1-k \eta^{-1}}
$$

and the inequality $\|x\|_{C([-h, T] ; E)} \leqslant R$ yields the estimate $\|G(x)\|_{C([-h, T] ; E)} \leqslant R$. Thus, the multi-operator $G$ maps a closed ball $B_{R}(0) \subset C([-h, T] ; E)$ into itself. By Theorem 2.1, the multi-operator $G$ possesses a fixed point, which by Theorem 3.1 is a mild solution of problem (1.1) -1.2 . The proof is complete.

\section{BIBLIOGRAPHY}

1. M.S. Afanasova, G.G. Petrosyan. On the boundary value problem for functional-differential inclusion of fractional order with general initial condition in a Banach space // Izv. VUZov. Matem. 63:9, 3-15 (2019). [Russian Math. (Izv. VUZov). 63:9, 1-12 (2019).]

2. G.G. Petrosyan, M.S. Afanasova. On the Cauchy problem for fractional differential inclusion with a nonlinear boundary condition // Vestnik Voronezh. Gosud. Univ. Ser. Fiz. Matem. 1, 135-151 (2017). (in Russian). 
3. M. Afanasova, Y. Ch. Liou, V. Obukhoskii, G. Petrosyan. On controllability for a system governed by a fractional-order semilinear functional differential inclusion in a Banach space // J. Nonl. Convex Anal. 20:9, 1919-1935 (2019).

4. J. Appell, B. Lopez, K. Sadarangani. Existence and uniqueness of solutions for a nonlinear fractional initial value problem involving Caputo derivatives // J. Nonl. Var. Anal. 2, 25-33 (2018).

5. I. Benedetti, V. Obukhovskii, V. Taddei. On generalized boundary value problems for a class of fractional differential inclusions // Fract. Calc. Appl. Anal. 20, 1424-1446 (2017).

6. K.-J. Engel, R. Nagel. A short course on operator semigroups. Springer, New York (2006).

7. R. Gorenflo, A.A. Kilbas, F. Mainardi, S.V. Rogosin. Mittag-Leffler functions, related topics and applications. Springer-Verlag, Berlin (2014).

8. R. Hilfer. Applications of fractional calculus in physics. World Scientific, Singapore (2000).

9. M. Kamenskii, V. Obukhovskii, P. Zecca. Condensing multivalued maps and semilinear differential inclusions in Banach spaces. Walter de Gruyter, Berlin (2001).

10. M. Kamenskii, V. Obukhovskii, G. Petrosyan, J.C. Yao. On semilinear fractional order differential inclusions in Banach spaces // Fixed Point Theory. 18:1, 269-292 (2017).

11. M. Kamenskii, V. Obukhovskii, G. Petrosyan, J.C. Yao. Boundary value problems for semilinear differential inclusions of fractional order in a Banach space // Appl. Anal. 97:4, 571-591 (2018).

12. M. Kamenskii, V. Obukhovskii, G. Petrosyan, J.C. Yao. On approximate solutions for a class of semilinear fractional-order differential equations in Banach spaces // Fixed Point Theory Appl. 28:4, 1-28 (2017).

13. M. Kamenskii, V. Obukhovskii, G. Petrosyan, J.C. Yao. Existence and approximation of solutions to nonlocal boundary value problems for fractional differential inclusions // Fixed Point Theory Appl. id 2 (2019).

14. T.D. Ke, N.V. Loi, V. Obukhovskii, Decay solutions for a class of fractional differential variational inequalities // Fract. Calc. Appl. Anal. 18, 531-553 (2015).

15. T.D. Ke, V. Obukhovskii, N.C. Wong, J.C. Yao. On a class of fractional order differential inclusions with infinite delays // Appl. Anal. 92, 115-137 (2013).

16. A.A. Kilbas, H.M. Srivastava, J.J. Trujillo. Theory and applications of fractional differential equations. Elsevier, Amsterdam (2006).

17. F. Mainardi. On the initial value problem for the fractional diffusion-wave equation // in "Waves and stability in continuous media". Proceedings VII WASCOM, Bologna (1993). World Scientific, Singapore 246-251 (1994).

18. V. Obukhovskii, B. Gelman. Multivalued maps and differential inclusions. Elements of theory and applications. World Scientific, Singapore (2020).

19. I. Podlubny. Fractional differential equations. Academic Press, San Diego (1999).

20. V.E. Tarasov. Fractional dynamics. Applications of fractional calculus to dynamics of particles, fields and media. Springer, Berlin (2010).

21. Z. Zhang, B. Liu. Existence of mild solutions for fractional evolution equations // Fixed Point Theory. 15:1, 325-334 (2014).

22. Y. Zhou, F. Jiao. Existence of mild solutions for fractional neutral evolution equations // Comput. Math. Appl. 59:3, 1063-1077 (2010).

23. Y. Zhou. Fractional evolution equations and inclusions: analysis and control. Academic Press, London (2016).

Garik Gagikovich Petrosyan,

Voronezh State University of Engineering Technologies,

Revolutsii av. 19,

394036, Voronezh, Russia

E-mail: garikpetrosyan@yandex.ru 